\title{
Radio Emission Properties of Millisecond Pulsars
}

\author{
Michael Kramer \\ Max-Planck-Institut für Radioastronomie, Bonn, Germany \\ University of Manchester, Jodrell Bank Observatory, $U K$ \\ Kiriaki M. Xilouris \\ Department of Astronomy, University of Virginia, Charlottesville, USA
}

\begin{abstract}
We report the most recent progress in understanding the emission properties of millisecond pulsars.
\end{abstract}

\section{Introduction - Duo quum faciunt idem, not est idem. ${ }^{1}$}

Through intensive research for almost two decades, it has been well established, both in theory and observation, that millisecond pulsars (MSPs) are the end product of mass accretion in binary systems. As MSPs emerge in the radio universe having been given a second chance in life, they are surrounded by magnetospheres which are several orders of magnitude more compact than those of slower rotating pulsars. Inferred magnetic fields close to the surface of MSPs are 3 to 4 orders of magnitude weaker than in normal pulsars while charges at these regions experience an accelerating potential similar to that of normal pulsars. The impact of the different environment on the emission process in MSP magnetospheres has been a question addressed already shortly after the discovery of a first few such sources.

With the plethora of MSPs detected over the years, a significant sample became available to us, enabling a better understanding of not only MSPs (as radio sources and tools) but slower rotating (normal) pulsars as well. In the following, we will concentrate on recent progress, referring to Kramer et al. (1998, Paper 1) on spectra, pulse shapes and beaming fraction; Xilouris et al. (1998, Paper II) on polarimetry of 24 MSPs; Sallmen (1998) and Stairs et al. (1999) on multi-frequency polarimetry; Ioscano et al. (1998) on spectra of Southern MSPs; Kramer et al. (1999b, Paper III) on multi-frequency evolution; and Kramer et al. (1999a, Paper IV) on profile instabilities of MSPs; but see also the following contributions by Kuzmin \& Losovsky and Soglasnov.

\section{Single Pulses vs. Average Profile Studies}

Single pulse observations still remain the only tool available to address some fundamental questions listed below. They are, however, still technically challenging and the number of observations described in the literature are scarce. In total, data for only three sources describing $180 \mathrm{~min}$ of observations have been

\footnotetext{
${ }^{1}$ If two do the same, it is not the same.
} 
presented, i.e. PSRs B1937+21, B1534+12 and J0437-4715 (e.g. Sallmen 1998, Cognard et al. 1996, Jenet et al. 1998 and references therein). The results can be summarized in the statement that based on the single pulses studied, one cannot distinguish between a millisecond or slowly rotating pulsar. More observations are required to further investigate pulse fluctuations (e.g. stabilization processes), the short-term structure (e.g. how it relates to microstructure) and in particular the polarization characteristics in detail. For the time being, we investigate the wealth of information already provided by average profile studies.

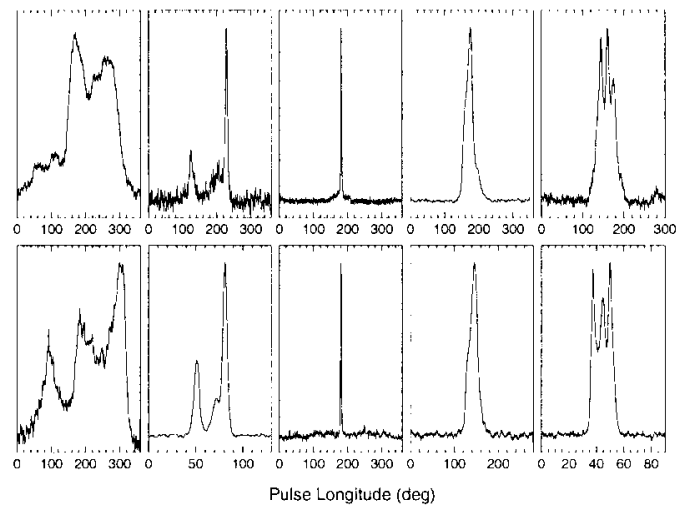

Figure 1. Pulse profiles for a selected sample of MSPs and normal pulsars (Paper I and EPN database). Note the similarity and make a guess which is which! See footnote ${ }^{2}$ for the solution.

\section{Flux Density Spectra and Radio Luminosity}

Prior to the investigations leading to Paper I it was commonly believed that the spectra of millisecond pulsars were steeper than those of normal pulsars. We demonstrated in Paper I that the distribution of spectral indices for MSPs is in fact not significantly different, finding an average index of $-1.76 \pm 0.14$ (Paper III). The initial impression was due to a selection effect, since the first MSPs were discovered in previously unidentified steep spectrum sources, as it was later pointed out by Toscano et al. (1998). Consequently, the number of MSPs to be discovered in high-frequency surveys was underestimated. The predictions for searches at frequencies as high as $5 \mathrm{GHz}$ appear even more favourable in light of the latest results presented in Paper III. These suggest that most spectra can be represented by a simple power law, i.e. clear indications for a steepening at a few $\mathrm{GHz}$ as known from normal pulsars are not seen. Extending the data to lower frequencies (see Paper III; Kuzmin \& Losovsky, next contribution), evidence for spectral turn-overs were not found.

Bailes et al. (1997) pointed out that isolated MSPs are less luminous than those in binary systems, pointing towards a possible relation between radio luminosity and birth scenarios. We have compared a distance limited sample

\footnotetext{
${ }^{2}$ Upper row: MSPs (PSRs J0218+4218, J0621+1001, B1534+12, J1640+2224, J1730-2304), lower row: normal pulsars (PSRs B1831-04, B2045-16, B2110+27, B2016+28, B1826-17)
} 

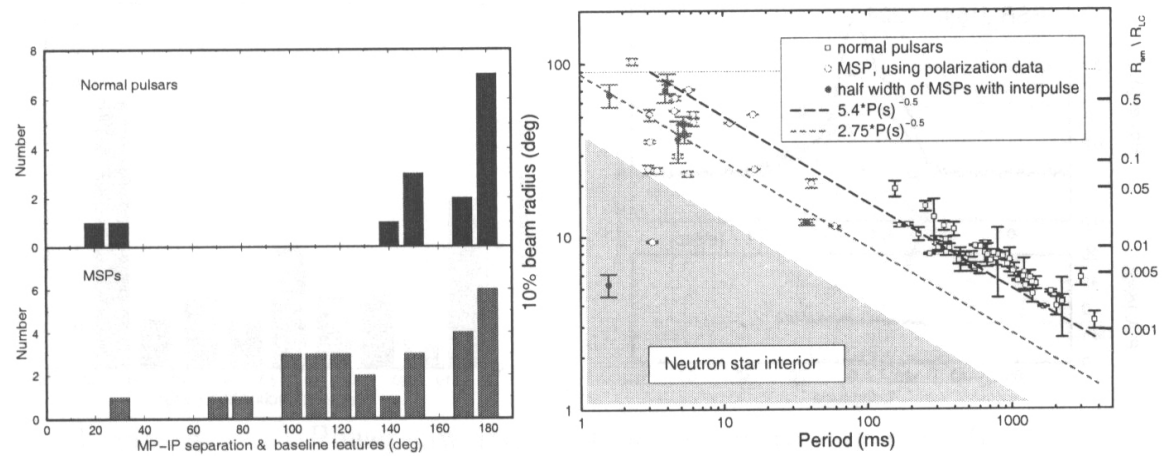

Figure 2. a) Location of additional pulse features across the pulse period for normal pulsars and MSPs. b) The beam radius, $\rho$, for normal pulsars and MSPs. MSPs do not follow the scaling law of normal pulsars (here Gould 1994) but their beaming fraction is much smaller. For MSPs with interpulses an "inner" relationship is indicated.

of normal pulsars and MSPs and came to a similar result with the MSPs as a whole appearing as weaker sources than normal pulsars.

\section{Pulse Profiles - Complexity, Interpulses and Beaming Fraction}

It was also believed that MSP profiles are more complex than those of normal pulsars. Using a large uniform sample of profiles for fast and slowly rotating pulsars, we showed in Paper I that the apparent larger complexity is due to the (typically) larger duty cycle of MSPs. As a result we see "blown-up" profiles which make it easier to see detailed structure. In fact, blown-up normal pulsar profiles show very similar structure. A quantitative proof is given in Paper I, while Fig. 1 provides an illustration of this effect.

Despite this apparent similarity, there is a profound difference betweent MSP profiles and those of normal pulsars! Additional pulse features like interpulses, pre- or post-cursor are much more common for MSPs. While only $\sim 2 \%$ of all normal pulsars are known to show such features, we detect them for more than $30 \%$ of all (field) MSPs. They also appear at apparently random positions across the pulse period in contrast to normal pulsars (Fig. 2a). Their frequent occurrence and location makes one wonder - given the similarity of the main pulse shapes otherwise - whether these components are of the same origin as the main pulse profile or whether other sources of emission (e.g. outer gaps) are responsible (see Paper II). Other possibilities involve an interpretation first put forward for some young pulsars by Manchester (1996), who interpreted some interpulses as the results of cuts through a very wide cone. This is an interesting possibility also for MSPs, since their beam width appears to be much smaller than predicted from the scaling law derived for normal pulsars. The beam width of normal pulsars, $\rho$, i.e. the pulse width corrected for geometrical effects (see 

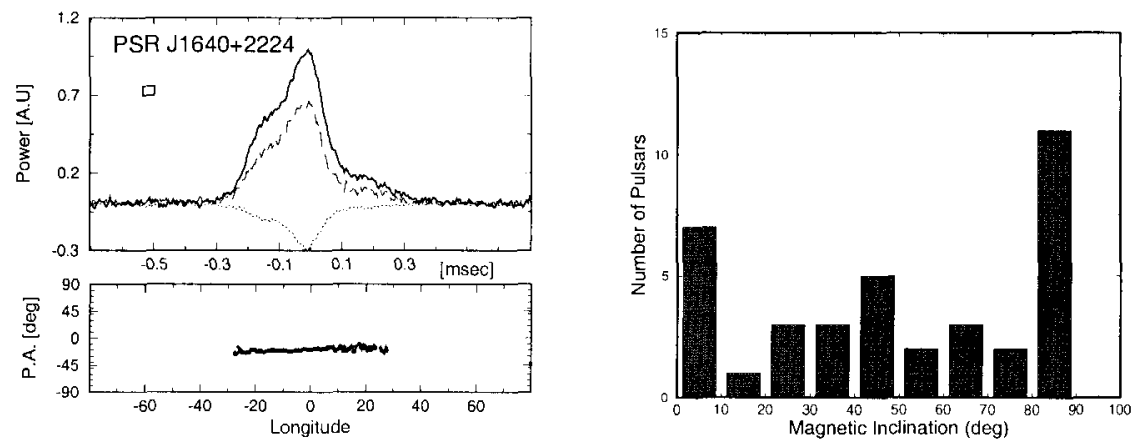

Figure 3 . a) PSR J1640+2224 as an example for a MSP exhibiting a flat PA swing, b) distribution of magnetic inclination angles derived from RVM fits.

Gil et al. 1984), follows a distinct $\rho \propto P^{-0.5}$-law (e.g. Rankin 1993, Kramer et al. 1994, Gould 1994). Using polarization information to determine the viewing geometry and also applying statistical arguments, we calculated $\rho$ (at a $10 \%$ intensity level) for MSPs in Paper I. We showed that they are not only much smaller than the extrapolation of the known law to small periods, but that under the assumption of dipolar magnetic fields ... the emission of some MSPs seems to come even from within the neutron star - a really disturbing result! While we discuss the possibility of non-dipolar fields and the used polarization information below, one explanation would be that (perhaps below a critical period) the emission beam does not fill the whole open field line region ("unfilled beam"). The situation improves somewhat when we consider the additional pulse features as regular parts of the pulse profile (Fig. 2b). In fact, those MSPs with interpulses may indicate an additional inner scaling parallel to that known for normal pulsars, which could be a result of unfilled beams. We close this section by pointing out that the much smaller beam width has consequences for population studies, which usually utilize the $\rho-P$ scaling as found for normal pulsars. The failure of this law leads to an overestimated beaming fraction and an underestimation of the birth rate of recycled pulsars (see Paper I).

\section{Polarization Properties}

The radio emission of MSPs shows all polarization features known from normal pulsars, i.e. circular polarization which is usually associated with core components, linear polarization which is usually associated with cone components, and also orthogonal polarization modes (see Paper II, Sallmen 1998, Stairs et al. 1999). Despite the qualitative similarities, the position angle (PA) swing is often strikingly different. While normal pulsars show typically a S-like swing, which is interpreted within the rotating vector model (RVM; Radhakrishnan \& Cooke 1969), the PAs of many MSPs often appear flat (see e.g. Fig. 3a). This could be interpreted in terms of non-dipolar fields, but Sallmen (1998) noted that larger beam radii lead to a larger probability for outer cuts of the emission 

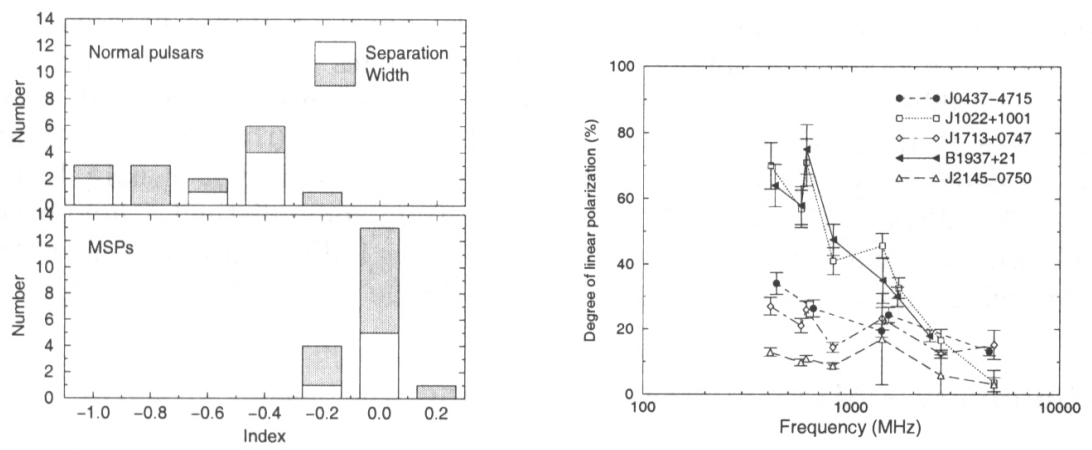

Figure 4. a) Power law index of profile narrowing with frequency (see Paper III for details), b) degree of polarization for MSPs.

cones, i.e. flatter PA swings according to the RVM. Although one should bear in mind the limitations of the $\rho$-scaling law and another caveat discussed later, this interpretation justifies the geometrical interpretation of the data, which is supported by the results of Hibschman (these proceedings). Magnetic inclination angles derived from RVM fits are important for binary evolution models and determinations of the companion mass (Fig. 3b).

\section{Frequency Evolution}

The radio properties of normal pulsars show a distinct frequency evolution, i.e. with increasing frequency the profile narrows, outer components tend to dominate over in ner ones, and the emission depolarizes. The emission of MSPs, which at intermediate frequencies tends to be more polarized than that of normal pulsars (Paper II), also depolarizes at high frequencies (Fig. 4b; Paper III). Simultaneously, the profile width hardly changes or remains constant (see Fig. 4a, Paper III; Kuzmin \& Losovsky, these proceedings). This puts under test attempts to link both effects to the same physical origin (i.e. birefringence). In fact, many profiles also exhibit the same shape at all frequencies, while others evolve in an unusual way, i.e. the spectral index of inner components is not necessarily steeper, so that a systematic behaviour as seen for normal pulsars is hardly observed. This can be understood in terms of a compact emission region, an assumption further supported by a simultaneous arrival of the profiles at all frequencies. We emphasize that we have not detected any evidence for the existence of non-dipolar fields in the emission region (Paper III).

\section{Profile and Polarization Instabilities}

The amazing stability with time of MSP profiles has enabled high precision timing over the years. However, in Paper IV we discussed the surprising discovery that a few MSPs do show profile changes caused by an unknown origin. Tho time scales of these profile instabilities are inconsistent with the known mode-changing. In particular, PSR J1022+1001 exhibits a narrow-band profile variation never seen before (Paper IV), which could, however, be the result of 
magnetospheric scintillation effects described by Lyutikov (these proceedings). With the pulse shape the polarization usually changes as woll, and hence this effect is possibly related to phenomena which we discovered in Paper II. Some pulsars like PSR J2145-0750 (Paper II) or PSR J1713+0747 (Sallmen 1998) show occasionally a profile which is much more polarized than usual. In the case of PSR J2145-0750, the PA also changes from some distinct (though not S-like) swing to some very flat curve. This is a strong indication that some of the flat PA swings discussed above may not be of simple geometrical origin alone.

\section{Summary - MSPs in 2000 and Beyond}

While we have had to be necessarily brief in reviewing MSP properties, we direct the interested reader to the extensive studies of MSPs presented in the quoted literature. We summarize here our point of view: MSPs emit their radio emission by the same mechanism as normal pulsars. Some distinct differences may originate from the way they were formed, but most observed features can be explained by very compact magnetospheres. Our data can be explained without any need to invoke deviations from dipolar field lines, although a large number of open questions remain. We need more polarization information at higher frequencies and, in particular, single pulse studies. These will allow us to study the formation of the profile and its stability, to see if the additional pulse features are distinct from the main pulse, and how the polarization modes behave under the magnifying glass of the blown-up MSP profiles. There are exciting years to come!

Acknowledgments. We are very grateful to all the people involved in the studies of MSPs at Bonn, i.e. Don Backer, Fernando Camilo, Oleg Doroshenko, Alexis von Hoensbroech, Axel Jessner, Christoph Lange, Dunc Lorimer, Shauna Sallmen, Norbert Wex, Richard Wielebinski and Alex Wolszczan.

\section{References}

Bailes, M., Johnston, S., Bell, J. F., et al. 1997, Ap.J, 481, 386

Cognard, I., Shrauner, J., Taylor, J. H., \& Thorset, S. E. 1996, ApJ, 457, 81

Gil, J., Gronkowski, P., \& Rudnicki, W. 1984, A\&A, 132, 312

Gould, D.M. 1994, PhD thesis, University of Manchester

Jenet, F., Anderson, S., Kaspi, V., et al., 1998, Ap.J, 498, 365

Kramer M., Xilouris K. M., Lorimer D. R., et al. 1998, ApJ, 501, 270 (Paper I)

Kramer M., Xilouris K. M., Camilo F., et al. 1999a, ApJ, 520, 324 (Paper IV)

Kramer M., Lange, Ch., Lorimer, D.R., et al. 1999b, ApJ, 526, 975 (Paper III)

Manchester, R.N. 1996, in Proc of IAU Colloq. 160, ASP Conf. Series, p. 193

Radhakrishnan, V., \& Cooke, D.J., 1969, ARA\&A, 32, 591

Rankin, J.M. 1993, Ap.J, 405, 285

Sallmen, S. 1998, PhD thesis, University of California at Berkeley

Stairs ,I. H., Thorsett, S. E., \& Camilo, F. 1999, ApJS, 123, 627

Toscano, M., Bailes, M., Manchester, R.N., \& Sandhu, J. 1998, Ap.J, 506, 863

Xilouris, K. M., Kramer, M., Jessner, A., et al. 1998, ApJ, 501, 286 (Paper II) 